\title{
Synthesis of $\operatorname{ReN}_{3}$ Thin Films by Magnetron Sputtering
}

\author{
G. Soto, ${ }^{1,2}$ H. Tiznado, ${ }^{1}$ W. de la Cruz, ${ }^{1}$ and A. Reyes ${ }^{1}$ \\ ${ }^{1}$ Universidad Nacional Autónoma de México, Centro de Nanociencias y Nanotecnología, Km 107 Carretera Tijuana-Ensenada, \\ 22800 Ensenada, BCN, Mexico \\ ${ }^{2}$ CNyN-UNAM, P.O. Box 439036, San Ysidro, CA 92143-9036, USA
}

Correspondence should be addressed to G. Soto; gerardo@cnyn.unam.mx

Received 9 October 2013; Revised 26 March 2014; Accepted 3 April 2014; Published 18 June 2014

Academic Editor: Roger Narayan

Copyright (C) 2014 G. Soto et al. This is an open access article distributed under the Creative Commons Attribution License, which permits unrestricted use, distribution, and reproduction in any medium, provided the original work is properly cited.

In this work $\mathrm{ReN}_{x}$ films were prepared by reactive magnetron sputtering at room temperature and deposited on a silicon wafer. It was found that the diffractograms of the nitrogen-rich rhenium film are consistent with those produced by high-pressure hightemperature methods, under the assumption that the film is oriented on the substrate. Using density functional calculations it was found that the composition of this compound could be $\mathrm{ReN}_{3}$, instead of $\mathrm{ReN}_{2}$, as stated on previous works. The ReN $\mathrm{N}_{3}$ compound fits in the Ama2 (40) orthorhombic space group, and due to the existence of $\mathrm{N}_{3}$ anions between Re layers it should be categorized as an azide. The material is exceptionally brittle and inherently unstable under indentation testing.

\section{Introduction}

In recent years, reports on the synthesis of novel materials by high-pressure and high temperature (HPHT) methods have become common. The use of extreme pressures and temperatures, combined with compositional variables, provides an opportunity to synthesize uncommon materials and/or to tune the physical properties of materials for a wide range of applications [1]. HPHT methods were pioneered by researchers looking to synthesize diamonds, but now are often applied to discover materials that might have mechanical, chemical or thermal resistance comparable to those of diamond. Many transition metal nitrides are poised as promising hard materials principally for machining of ferrous alloys. Using HPHT methods, novel nitrides of heavy metals such as platinum, osmium, and iridium [2, 3] have been synthesized. Due to the observed high bulk modulus of these materials, some HPHT studies have sought to discover novel nitrides of heavy metals. An example of this effort is the work of Kawamura et al., who recently published an article describing the HPHT synthesis of $\mathrm{ReN}_{2}$ [4].

In direct competition with HPHT methods for the synthesis of artificial diamond are methods using plasma [5]. These have been successful in terms of the quality and purity of the obtained diamond, with the added advantage that these materials can be applied as coatings directly from the synthesis. Similarly, the plasma methods are used to discover alternative materials that can replace diamond. The success of plasmas for producing new materials originates from the high reactivity obtained by breaking molecular bonds after multiple ionization events. Using laser ablation experimentsa very energetic plasma method-it was possible to grow films composed of $\mathrm{AuN}_{x}$ [6], $\mathrm{PtN}_{x}$ [7], and $\mathrm{RuN}_{x}$ [8]. The main limitations of plasma synthesis are the low growth rates and the textured crystalline orientations, which make the obtained materials not easy to characterize.

Recently, we published an article describing the synthesis of rhenium nitride using a plasma method [9]. We were successful in describing the experimental conditions and the results when nitrogen is incorporated in low concentrations. Basically, the $\operatorname{ReN}_{x}(x \leq 1)$ consists of a solid solution of nitrogen incorporated into the octahedral interstices of a rhenium lattice. However in this work we failed in interpreting the data when nitrogen is present in high concentrations $(x \geq$ 1). Recently, Kawamura et al. reported the HPHT formation of a rhenium-nitrogen compound, and they conclude to have synthesized $\mathrm{ReN}_{2}$, with structure similar to $\mathrm{MoS}_{2}$ [4]. The report of Kawamura et al. was very useful in resolving the 
structure of our films in the nitrogen-rich condition. As a result, the objective of this report is to describe the type of compound that is produced when a rhenium target is deposited by DC sputtering in an excess of nitrogen, that is, in target-poisoned state. As a clarification, the target poisoning means that the compound is formed not only on the substrate, as desired, but also on the sputter target, which results in a significantly reduced sputter yield and, therefore, a reduced deposition rate [10]. This regime of deposition is seldom used for technological applications, but it can be used for the preparation of novel compounds.

\section{Method}

2.1. Thin Film Preparation and Characterizations. The rhenium nitride films were grown by reactive DC sputtering. The experiment was carried out in a custom-made ultrahigh vacuum (UHV) deposition system. It consists of three stainless steel chambers: sample loading, deposition, and analysis. The analysis chamber is a PHI-548 system equipped with X-ray photoelectron (XPS) and Auger electron (AES) spectroscopies, and it operates at $10^{-10}$ Torr, achieved by means of an ion pump. The sample loading and deposition chambers are independently evacuated to pressures $10^{-8}$ Torr or lower by using a $130 \mathrm{~L} \mathrm{~s}^{-1}$ turbomolecular pump. A $2.5 \mathrm{~cm}$ diameter Re target (99.9\%) is positioned in a magnetron gun, $10 \mathrm{~cm}$ above a (111)-silicon substrate. A mechanized rod is used to transfer the sample between chambers, which are isolated by gate valves. With this setup the deposited films can be analyzed without exposure to the outside atmosphere. The rhenium nitride films were deposited at room temperature at different $\mathrm{N}_{2}$ pressures in the following manner. In the deposition chamber, a mass-flow controller fed $\mathrm{N}_{2}$ (ultrahigh purity grade) at increasing flow rates to set pressures ranging from 3 mTorr $\left(1 \mathrm{~mL} \mathrm{~min}^{-1}\right)$ to $180 \mathrm{mTorr}\left(35 \mathrm{~mL} \mathrm{~min}^{-1}\right)$. After setting the $\mathrm{N}_{2}$ pressure, an additional $5 \mathrm{~mL} \mathrm{~min}^{-1}$ flow of $\mathrm{Ar}$ (ultrahigh purity grade) was allowed into the chamber. The samples were grown at 120 Watts for 50 minutes, after that they were immediately transferred to the analysis chamber for XPS and AES measurements. A reference sample, metallic $\mathrm{Re}$, was deposited in the absence of $\mathrm{N}_{2}$. The assessment of nitride formation was performed by comparing core-level energy shifts in the XPS lines to the metallic Re sample. The XPS measurements used an $\mathrm{Al} \mathrm{K}_{\alpha}(h v=1486.6 \mathrm{eV}) \mathrm{X}$-ray nonmonochromatic source. The X-ray diffraction (XRD) patterns were collected ex situ in a Philips X'pert diffractometer using the $\mathrm{Cu} \mathrm{K}_{\alpha}$ line with a wavelength of $\lambda=0.154 \mathrm{~nm}$. The film morphology and roughness were determined by atomic force microscopy using a NanoScope III, Veeco Instruments, in contact mode. The mechanical properties of the samples were measured using a TriboScope nanoindenter, Hysitron.

2.2. First Principle Calculations. Calculations were realized within the framework of density functional theory. We employed the full-potential linearized augmented plane wave method, as implemented in the Wien $2 \mathrm{k}$ code [11], using the generalized gradient approximation [12]. We used muffin-tin radii $\left(r_{\mathrm{mt}}\right)$ of $2.0 \mathrm{au}$ for $\mathrm{Re}$ atoms and of $1.0 \mathrm{au}$ for $\mathrm{N}$ and angular momenta up to $l=10.64 k$-points were used in the irreducible part of the Brillouin zone. Optimization of volume, $c / a(b=c t e), b / a(c=c t e)$, and internal atomic positions were performed recursively. Having arrived at the minimum energy structures, their volumes were varied around the equilibrium value, and their bulk moduli were obtained after fitting the Murnaghan equation of state to the calculated volume-energy data. The compound formation energy, $E_{A}$, from molecular nitrogen is computed as the difference between the ab initio total energies of $\mathrm{ReN}_{x}$ and those of Re metal and molecular $\mathrm{N}_{2}$ [13]. Consider

$$
E_{\mathrm{ReN}(A)}^{\prime}=\frac{E_{i}^{\mathrm{Tot}}-n E_{\mathrm{Me}-\text { bulk }}^{\mathrm{Tot}}-m(1 / 2) E_{\mathrm{N}_{2}}^{\mathrm{Tot}}}{m+n} .
$$

Here $n$ and $m$ are the number of metal and nitrogen atoms, respectively. From this definition, less positive values mean higher stability of the compound, relative to its spontaneous dissociation to $\mathrm{Re}$ and $\mathrm{N}_{2}$ constituents. Similarly, the formation energy from free atomic nitrogen, $E_{B}$, is computed as follows:

$$
E_{\mathrm{ReN}(B)}^{\prime \prime}=\frac{E_{i}^{\mathrm{Tot}}-n E_{\mathrm{Me}-\text { bulk }}^{\mathrm{Tot}}-m E_{\mathrm{N}}^{\mathrm{Tot}}}{m+n} .
$$

Because the molecular and atomic species cannot be treated directly within Wien $2 \mathrm{k}$, the $\mathrm{N}_{2}$ dimer was simulated by constructing a sufficiently large tetragonal unit cell (space group $P 4 / \mathrm{mmm}, a=4.50 \AA$, and $c=5.60 \AA)$, locating $\mathrm{N}_{2}$ in the vertices along the $z$-axis (N-positions $(0,0, \pm 0.0981))$ and performing the calculation for a single $k$-point at the origin of the first Brillouin zone. The nitrogen atomic species was carried out with analogous calculations for a nitrogen atom placed into the fcc unit cell ( Fm $3 m, a=15 \AA)$. To confirm that our calculations can be used for nitrogen-rich compounds, we performed equivalent calculations for $\mathrm{AgN}_{3}$. We have minimized the forces acting on all atoms. Our calculations agree with the experimental parameters for $\mathrm{AgN}_{3}$ and with previous calculations for that compound [14]. The results from these calculations are summarized in Table 1.

\section{Results}

3.1. Thin Film Deposition. The results for the deposition of $\operatorname{ReN}_{x}$ films $(x<1)$ were discussed in our previous work [15]. In this new work the same experimental setup and method are used for film preparations; however we will focus on the nitrogen-rich conditions, that is, target in poisoned state. The nitrogen-rich rhenium film was prepared at room temperature and a pressure of 180 mTorr $\mathrm{N}_{2}\left(F_{\mathrm{N}}=\right.$ $35 \mathrm{~mL} \min ^{-1} ; F_{\mathrm{N}}$ is the nitrogen flow), with an additional flow of $\mathrm{Ar}, F_{\mathrm{Ar}}=5 \mathrm{~mL} \mathrm{~min}{ }^{-1}\left(P_{\mathrm{Ar}} \sim 5 \mathrm{mTorr}\right)$. Figure 1 presents several XRD patterns of rhenium nitrides grown at different nitrogen flow rates. This figure is important to establish a comparison between compounds with low and high nitrogen contents. The interpretation of these diffractograms follows.

The peaks indicated with an asterisk $\left({ }^{*}\right)$ represent background from the silicon wafer substrate, which exhibits peaks at $28.40^{\circ}$ (111) and $58.85^{\circ}$ (222). The sample deposited with 
TABLE 1: Compound, structural parameters, unit cells, and DFT results of $\mathrm{ReN}_{2}$ and $\mathrm{ReN}_{3}$.

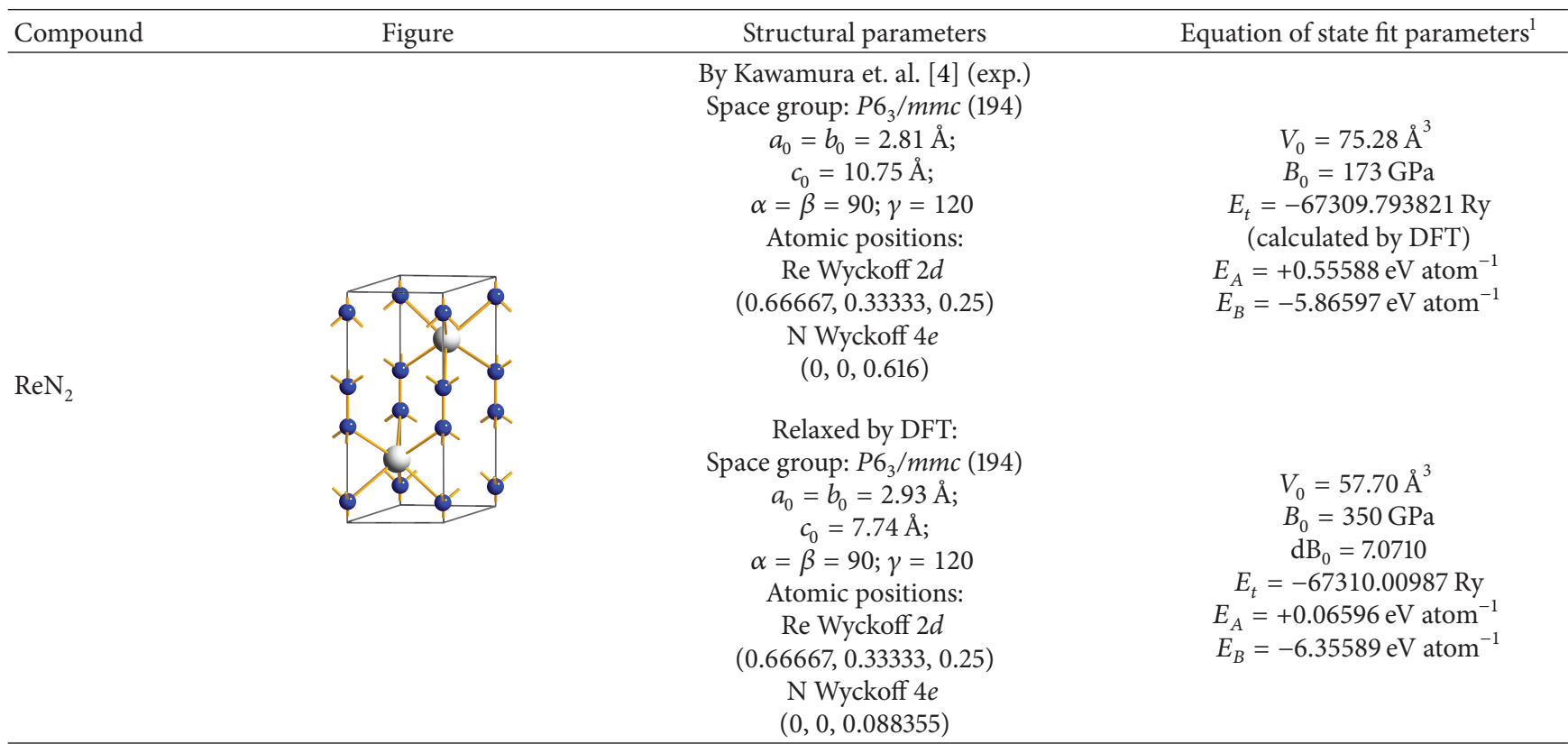

$\operatorname{ReN}_{3}$

Space group: $\mathrm{P}_{3} / \mathrm{mmc}$ (194)

$a_{0}=b_{0}=2.90 \AA ;$

$c_{0}=10.41 \AA$

$\alpha=\beta=90 ; \gamma=120$

Atomic positions:

Re Wyckoff $2 d$

$(0.66667,0.33333,0.25)$

$\mathrm{N}$ Wyckoff $4 e$

$(0,0,0.12365)$

$\mathrm{N}$ Wyckoff $2 a$ $(0,0,0)$

Space group: Am2a (40)

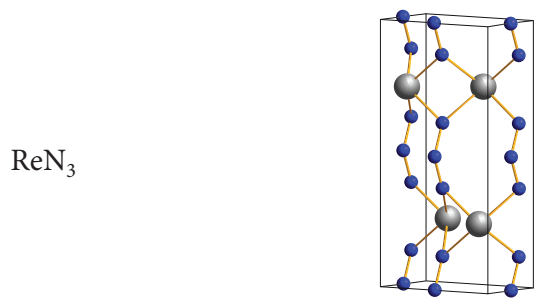

$a_{0}=10.06 \AA ; b_{0}=5.00 \AA$;

$c_{0}=2.95 \AA$;

$\alpha=\beta=\gamma=90$

Atomic positions:

Re Wyckoff $4 b$

$(0.25,0.66751,0.30367)$

N Wyckoff $8 c$

$(0.12255,0.00012,0.31473)$

$\mathrm{N}$ Wyckoff $4 a$

$(0,0,0.48985)$ ${ }_{1}^{1} E_{t}\left(\mathrm{Re}_{\mathrm{HCP}}=-31252.13779 \mathrm{Ry}\right) ; E_{t}\left(N_{2}=-219.04782 \mathrm{Ry}\right) ; E_{t}\left(N_{\text {Atomic }}=-108.81591 \mathrm{Ry}\right)$. Values obtained by DFT using the same level of theory than the ReN
compounds.

$F_{\mathrm{N}}=0$ and presented in Figure 1(a) shows three main diffraction peaks at $37.51^{\circ}, 40.4^{\circ}$, and $42.8^{\circ}$. These correspond to the diffraction planes (100), (002), and (101), respectively, of the hexagonal close-packed structure reported for pure $\mathrm{Re}$ in the powder diffraction file (PDF) number 89-2935. The (002) peak corresponds to a Re-Re interplanar spacing of $2.23 \AA$ in the $c$-direction. This pure-Re film is to some extent preferentially oriented, with (002)-Re parallel to (111)-Si. For the sample deposited with $F_{\mathrm{N}}=16 \mathrm{~mL} \mathrm{~min}^{-1}$, presented in Figure 1(b), the peaks corresponding to hexagonal Re were not present. Instead, wider peaks at approximately $38.45^{\circ}(\mathrm{A})$, $44.69^{\circ}(\mathrm{B}), 65.20^{\circ}(\mathrm{C})$, and $78.1^{\circ}(\mathrm{D})$ appear in the pattern. This pattern agrees with the face-centered cubic phase reported by Fuchigami et al. [16], with $a_{o}=4.03 \pm 0.03 \AA$. This pattern also agrees with a DFT computer-generated diffractogram (not shown here) for cubic $\operatorname{Re}_{4} \mathrm{~N}$, with $\mathrm{N}$ present in octahedral interstices. For higher values of $F_{\mathrm{N}}$, the positions of peaks A through $\mathrm{D}$ shift to lower angles as the $\mathrm{N} / \mathrm{Re}$ ratio within the films increases. The shift is more noticeable for the Apeak, which corresponds to the (111)-plane when referenced to the cubic phase. In Figure 1(c), this peak shifts to $38.16^{\circ}$ (corresponding to a Re-Re interplanar spacing of $2.35 \AA$ ) and then to $36.46^{\circ}$ in Figure $1(\mathrm{~d})$ (corresponding a Re-Re interplanar spacing of $2.46 \AA$ ). These shifts indicate that 


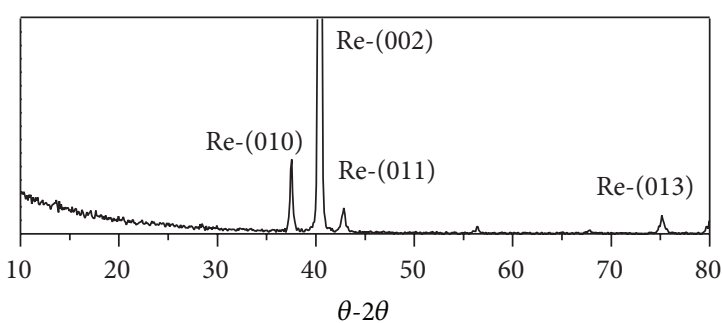

(a)

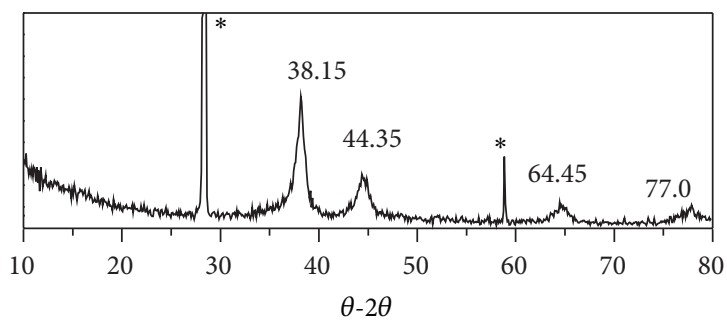

(c)

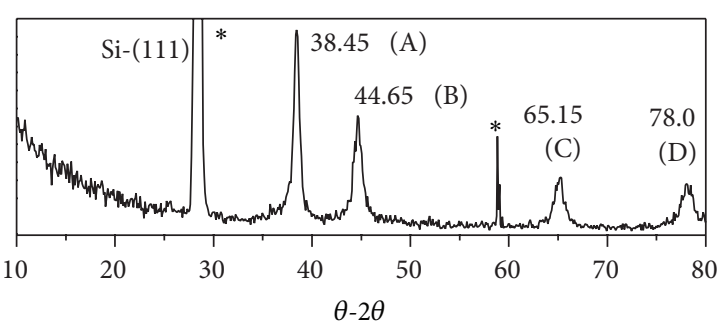

(b)

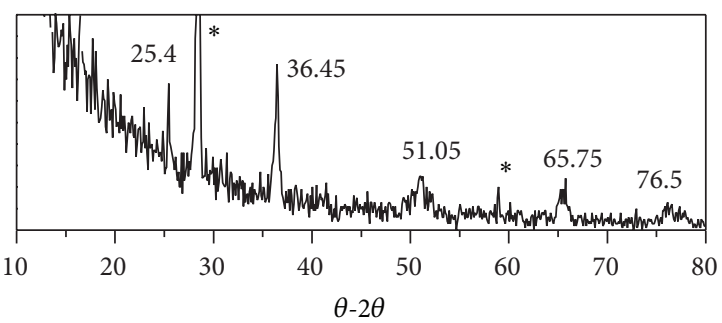

(d)

FIGURE 1: XRD patterns for DC-sputtered thin films: (a) $F_{\mathrm{N}}=0$; (b) $F_{\mathrm{N}}=16$; (c) $F_{\mathrm{N}}=20$; (d) $F_{\mathrm{N}}=30 \mathrm{~mL} \mathrm{~min}{ }^{-1}$.

the crystalline structure expands with increasing nitrogen content. Taking the most important diffraction peaks from Figure 1, in Figure 2 we show the dependence of interplanar distance on nitrogen flow rate during deposition. Additionally, in Figure 1(d), for the sample prepared with $F_{\mathrm{N}}=$ $35 \mathrm{~mL} \mathrm{~min}^{-1}$, two new broad peaks appear at approximately $51.02^{\circ}(\mathrm{E})$ and $65.44^{\circ}(\mathrm{F})$. These peaks cannot be indexed to a cubic phase and hereafter are related to a different arrangement of rhenium nitride, given their shape and position. In the same sample, some peaks were present at $16.45^{\circ}(110)$ and $25.47^{\circ}$ (210); these might be indexed to a hexagonal crystalline phase of $\mathrm{ReO}_{3}$, according to PDF number 40-1155. Keep in mind that the XRD measurements were taken ex situ, and then some amount of oxidation of samples is expected.

At the time that this film was synthesized, we conducted an exhaustive search trying to index this pattern to a known crystalline phase of rhenium compounds. We found that no phase described in the PDF database or in the reviewed literature for rhenium in the metallic, nitride, or oxide forms is in agreement with the diffraction pattern presented in Figure 1(d), except for the peaks at 16.45 and 25.7 related to the formation of $\mathrm{ReO}_{3}$. However, it is clear from Figure 2 that there is a noticeable expansion of the crystal lattice as a function of increasing nitrogen incorporation. The expansion occurs preferentially on the close-packed direction of the rhenium atoms, that is, along the (002)-direction when the hexagonal structure of metallic Re is taken as reference and along the (111)-direction when the cubic structure of $\operatorname{ReN}_{x}$ is taken as reference.

3.2. Nanoindentation Testing of Nitrogen-Rich Films. Figure 3 shows several load-unload displacement cycles for the $\mathrm{ReN}_{x}$ sample growth in nitrogen-rich conditions with applied load limits of 50,500 , and $1000 \mu \mathrm{N}$. The cycles consist of $5 \mathrm{~s}$ of an increasing ramp-up to the maximum load, $5 \mathrm{~s}$ of

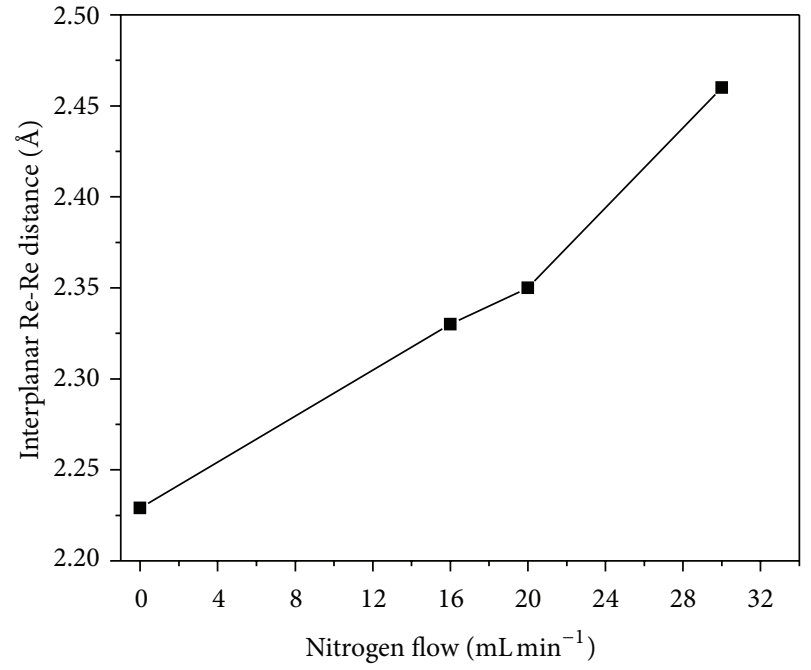

FIGURE 2: Interplanar Re-Re distance as a function of nitrogen flow during deposition.

sustaining the maximum load, $5 \mathrm{~s}$ of discharge, and $3 \mathrm{~s}$ of added acquisition at the contact force. It can be seen that this film shows a very peculiar behavior. For a typical sample during the ramp-up phase, when the indenter contacts the material, it is observed that the sample initially undergoes elastic deformation until the applied load reaches a critical value. When this critical value is exceeded, plastic deformation takes place, which is characterized by a sudden depth incursion or a "pop-in" [17]. The peculiar aspect of this material is the number of pop-ins observed in the loaddisplacement curve. As we can see from the inset of Figure 3, the elastic deformation is very small before the material experiences plastic deformations. These sudden insertions appear in the plot as vibrations of the Berkovich tip. The 
deformation is inherently unstable, occurring in bursts of highly localized strains [18]. There are also larger pop-in events that can be observed in the load-displacement curves, $\delta_{\text {exc }}$. Taken as a whole, the deformation is the sum of the small and large pop-in events that begin at infinitesimal loads. When a material has the tendency to fracture with very little or no detectable deformation beforehand, it is considered brittle. We can consider the current material to have perfectly brittle mechanical behavior because there is no elastic deformation. When the load is held constant, the plastic flow is evident because the penetration depth increases noticeably. Given that the film has a thickness of one micron (estimated by means of X-ray measurements) a creep of $100 \mathrm{~nm}$ is significant. Analyzing the initial portion of the unloading response gives an estimate of the elastic modulus of the indented material. As we can see, the initial slope of the unloading portion approaches zero, $d P / d H \rightarrow 0$, which means that this material is very soft. A detailed analysis of the raw data (difficult to detect at the scale pictured) shows extremely small "pop-out" events in the unloading portion of the curve. These "pop-outs" are attributed to the release of internal stresses generated during loading [19]. Some of the deformation is recovered by the pop-outs. The strangest thing is that this material fully recovers from its deformation after removal of the load. To be exact, the hysteresis curve returns to the initial point upon removal of the load, as seen in Figure 3. In experiments using the Berkovich tip as an AFM probe, it can be seen that no vestige of the indentations remains, as shown in Figure 4, which illustrates the film before and after indentation. We have no explanation for this behavior because, as previously stated, this is a brittle material. However, we speculate that this results from chemical changes (oxidation) in the sample rather than a recovery from mechanical deformations of $\mathrm{ReN}_{3}$.

\section{Discussion}

To discuss our results we want to use DFT calculations and the experimental result from the work of Kawamura et al. for a material that was assigned a $\mathrm{ReN}_{2}$ composition [4]. The interpretation of Kawamura was used as the starting point for our DFT calculations. In his article Kawamura explains a metathesis reaction between lithium nitride and rhenium pentachloride in a HPHT environment, which produces materials shaped like platelets. Kawamura provides powder XRD patterns according to Bragg-Brentano and Gandolfi geometries. All diffraction peaks were indexed by Rietveld analysis to a hexagonal $P 63 / m m c$ (194) space group, which is isostructural with $\mathrm{MoS}_{2}$ and similar to $\mathrm{ReB}_{2}$. The Rietveld analysis gave a lattice constant of $a=2.806 \AA, c=10.747 \AA$, and $V=75.28 \AA^{3}$ as well as an atomic coordinate of $\mathrm{N}(z)=0.616$. XPS analysis was used to confirm the Re-N bonding. The authors conclude that their synthesis produces $\mathrm{ReN}_{2}$. However the structural parameters differ from some theoretical calculations for rhenium-nitrogen compounds in $1: 2$ stoichiometric ratios [20-22]. While the reasons for the discrepancy were not clarified, it was assumed that this type of synthesis often results in the development of unusual

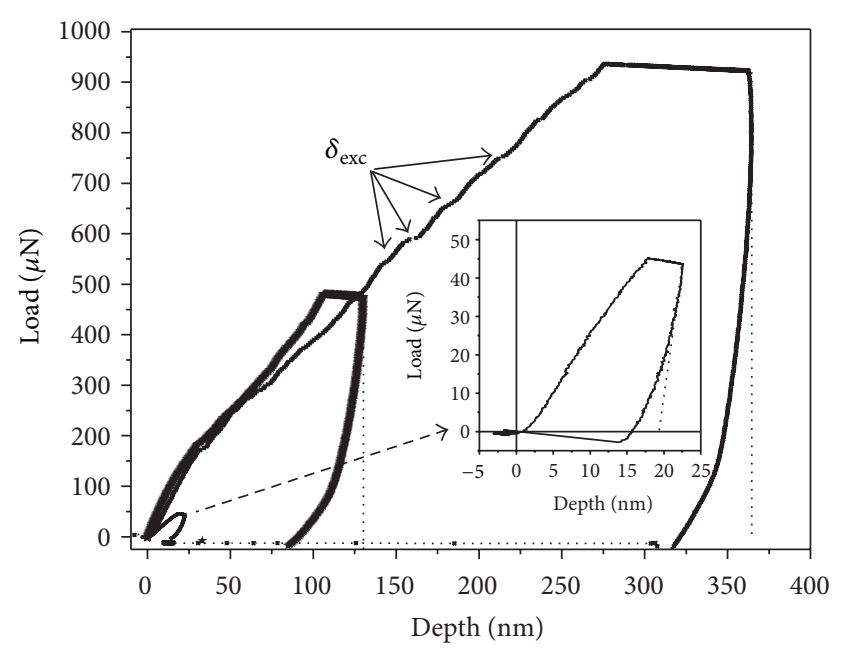

Figure 3: Three different load-displacement curves for the sputtered film, using a Berkovich diamond nanoindenter.

metastable phases. The unit cell and structural parameters proposed by Kawamura are given in Table 1 .

The X-ray pattern for the films growth by sputtering at pressure of 180 mTorr of $\mathrm{N}_{2}$ can be compared with the XRD pattern generated for structure proposed by Kawamura et al. [4]. That is shown in Figure 5, where the experimental diffraction is put side by side with computer-generated patterns from the structure described by Kawamura under particular conditions. Figure 5(a) is the experimental thin film pattern using the Bragg-Brentano geometry for the $\mathrm{Cu}-\mathrm{K}_{\alpha}$ radiation. Figure 5(b) presents the generated powder pattern for the Kawamura's structure using the $\mathrm{Cu}-\mathrm{K}_{\alpha}$ wavelength. Figure 5(c) presents only the peaks that can be associated with the experimental pattern. Figure 5(d) presents the diffractogram for the structure proposed by Kawamura with the assumption that this material is highly oriented in the $c$-direction using the Bragg-Brentano geometry; this pattern agrees with the data reported by Kawamura et al. in the inset of Figure 3 in [4]. It can be observed that the XRD pattern of the sputtered film can be indexed to the material synthesized by Kawamura et al. [4]. However, the film has a texture that is different from that observed by Kawamura. The texture is defined as the distribution of crystallographic orientations of a polycrystalline sample. In the film, the crystallographic orientations are not random but rather assume a preferred orientation of (010)-planes parallel to (111)-planes of the Si substrate. There is some contribution from (014)-planes, indicative of some tilt in the $c$-direction, and some contribution from (120)- and (124)-planes. Figure 6 presents a sketch illustrating these possibilities. In conclusion, the (010)-planes of the film are parallel to the (111)$\mathrm{Si}$ orientation, with slightly misoriented grains around this preferential orientation. As a consequence of this texture, there are missing reflections from the sample in the BraggBrentano geometry and to some extent weak counting rates. In contrast, the material synthesized by Kawamura is highly textured in the $c$-direction. We conclude that it is very likely that the material that was synthesized by the HPHT method 


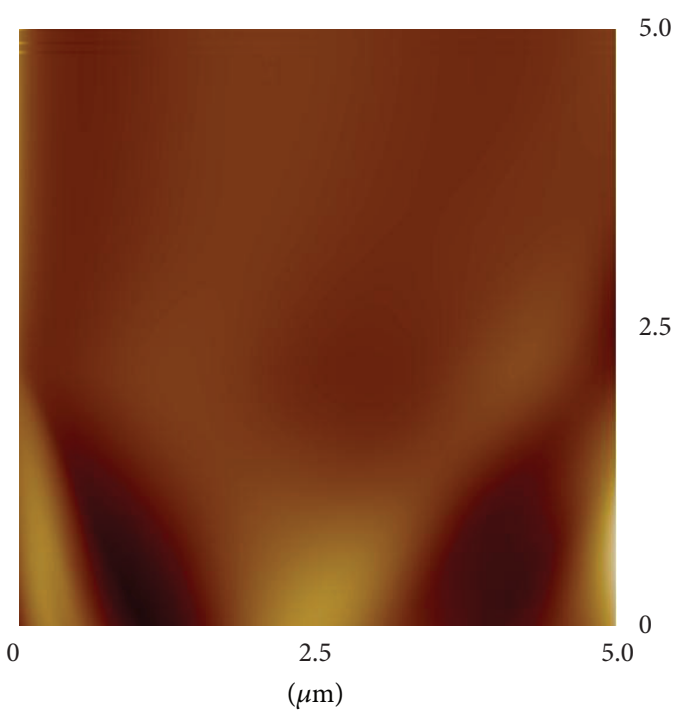

(a)

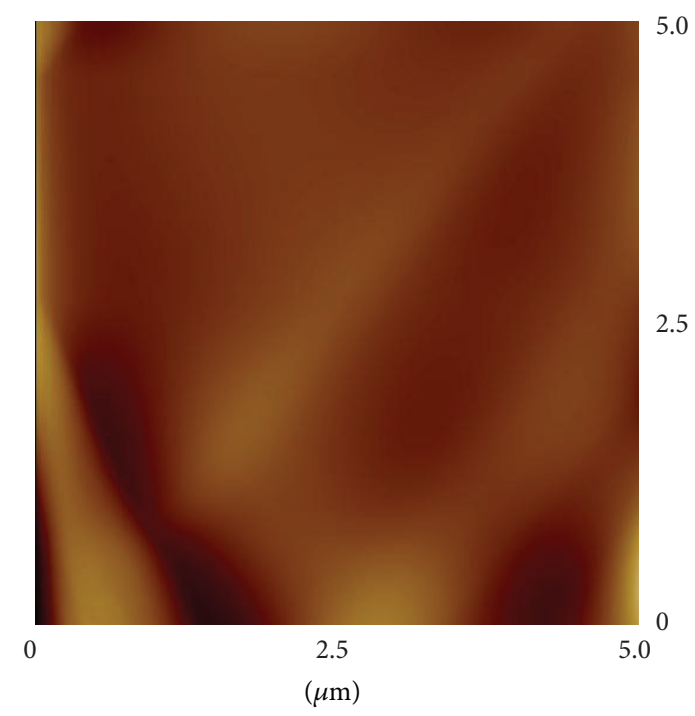

(c)

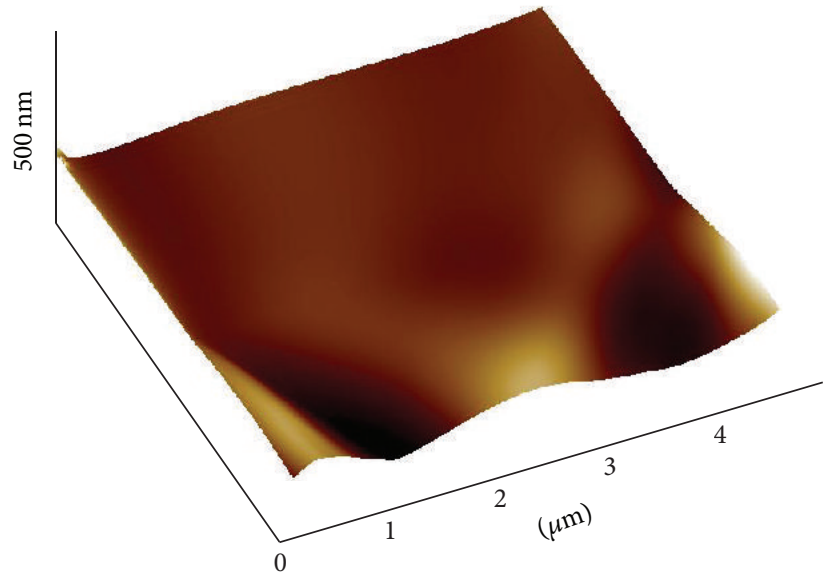

(b)

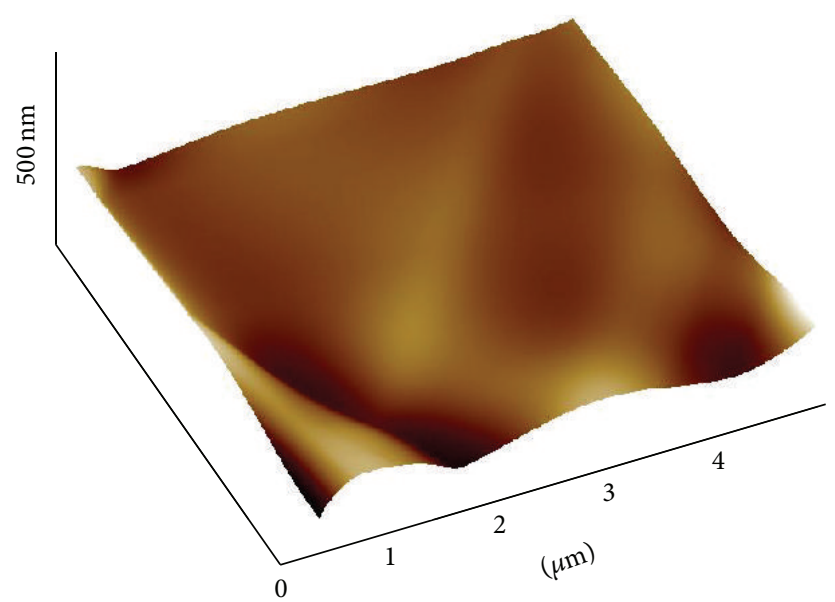

(d)

Figure 4: AFM images taken before ((a) and (b)) and after indentation ((c) and (d)). Although $\mathrm{ReN}_{3}$ is a brittle material, there are no traces of the indentation remaining in the surface.

has the same crystalline system as the material that was synthesized by the sputtering method.

To understand more deeply the nature of this compound, we performed DFT calculations using the structure proposed by Kawamura as starting point. The plot of total energy against volume is shown in Figure 7. The derivative $d E / d v$ at the volume given by the structural parameters reported by Kawamura is not zero. $d E / d v \neq 0$ implies that the system has not reached equilibrium. For any metastable point the cancelation of forces must be complete, being that an indispensable condition of metastability. Surprisingly, the structure proposed by Kawamura cannot be a metastable point. This implies that the structure proposed by Kawamura is not fully relaxed. This becomes evident since the energy of $\mathrm{ReN}_{2}$ at the points defined by Kawamura is high in comparison with the energy of the relaxed structure calculated by DFT; see Table 1 . In addition, the volume at the points defined by Kawamura is larger than the volume of the relaxed structure. It is unlikely that a structure that was synthesized under pressure would develop a metastable phase that is a "tensioned variation" of the relaxed structure. Very recently it was proposed, using DFT structural optimizations, that the $\mathrm{MoS}_{2}$-like structure of $\mathrm{ReN}_{2}$ will reform due to an rearrangement of the $\mathrm{N}_{2}$ dimers, in such a way that the hexagonal symmetry is lost [23]. Evidently, the diffraction pattern of the DFT-improved structure will not match the $\mathrm{X}$-ray diffraction reported by Kawamura and, therefore, will not match either of the diffractograms obtained in this work. 


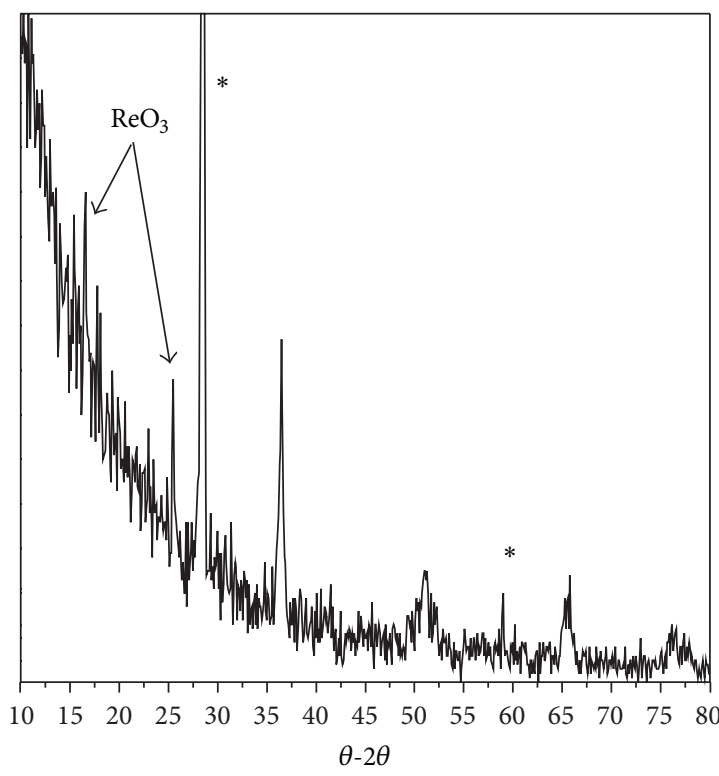

(a)

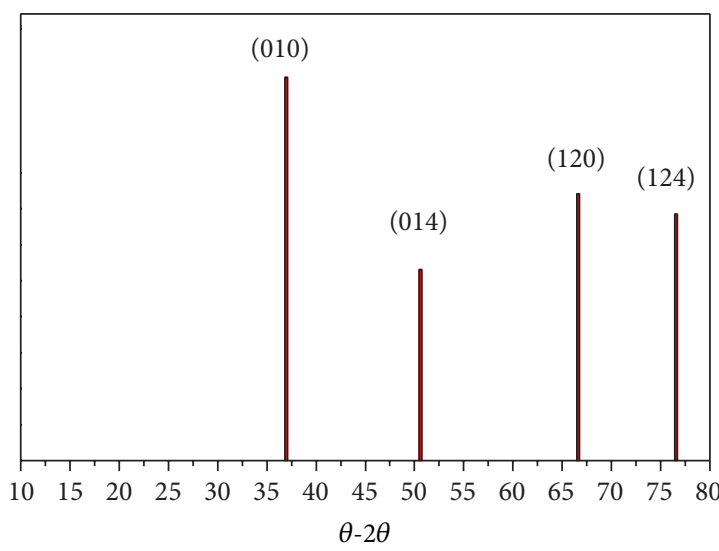

(c)

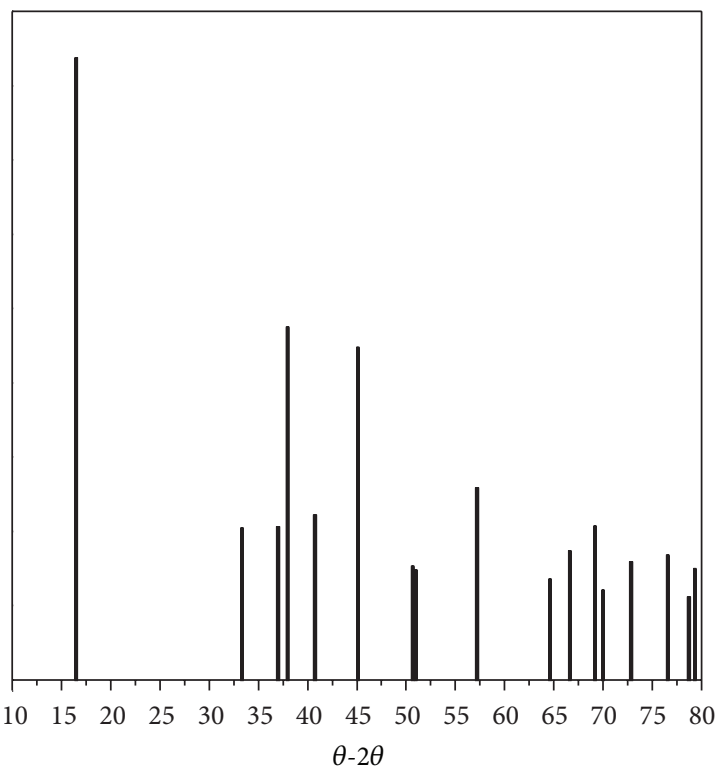

(b)

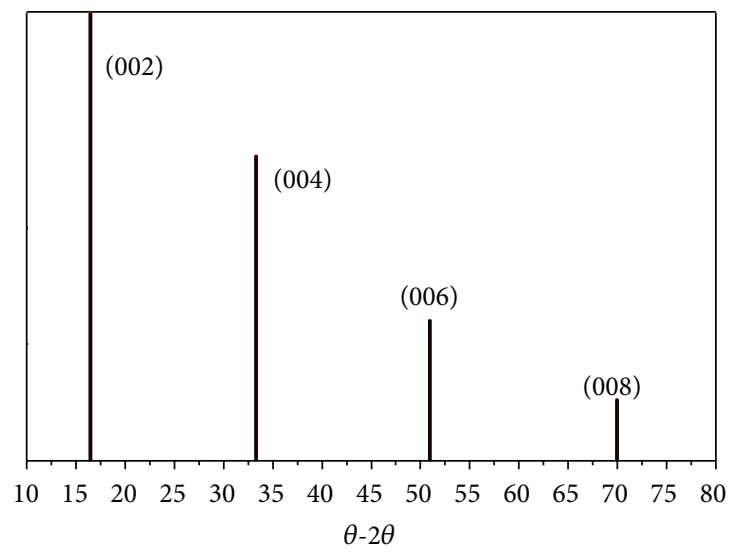

(d)

FiguRE 5: Comparison of the diffraction patterns for (a) experimental $\operatorname{ReN}_{x}$ film growth at $F_{\mathrm{N}}=35 \mathrm{~mL} \mathrm{~min}^{-1}$, (b) the powder pattern from the data of Kawamura et al., (c) diffractions from the data of Kawamura et al. that match the experimental pattern, and (d) the pattern reported by Kawamura et al. using the Braggs-Brentano geometry.

Additionally, in [23], the $\mathrm{MoS}_{2}$-like structure of $\mathrm{ReN}_{2}$ was found to be mechanically unstable according to the BornHuang criterion for a hexagonal structure because a negative value for the $\mathrm{C}_{44}$ elastic constant was found. A negative value for the $\mathrm{C}_{44}$ constant implies that the structure is tensioned and that the related strain tensor needs to be relaxed in the opposite direction. This is identical to our findings and is illustrated in Figure 7, where the relaxation of $c / a$ to lower ratios is a crucial step toward finding the lowest-energy point. In brief, the structure as it was proposed by Kawamura lacks certain features in the $c$-direction. The DFT calculations reveal that the empty space in $c$-direction cannot be justified by chemical bonds or by Van der Waals forces. With the goal of elucidating this irregularity, it was conjectured that there was sufficient space between the Re layers to insert $\left(\mathrm{N}_{3}\right)$ azide anions instead of $\left(\mathrm{N}_{2}\right)^{2}$-diazenide anions. The azide functional group is larger than the diazenide group; the larger anion could therefore match the interplanar spacing between the Re layers. Table 1 lists the structural parameters resulting from the DFT optimization of $\mathrm{ReN}_{3}$ constrained within the $P 63 / m m c$ (194) space group. It can be seen that the relaxed structure calculated by DFT for the $\mathrm{ReN}_{3}$ composition is a better match for the cell parameters given by Kawamura. This suggests that the compound of interest might be $\mathrm{ReN}_{3}$ rather than $\mathrm{ReN}_{2}$. However, the atomic positions of nitrogen and rhenium are highly constrained when the P63/mmc (194) 


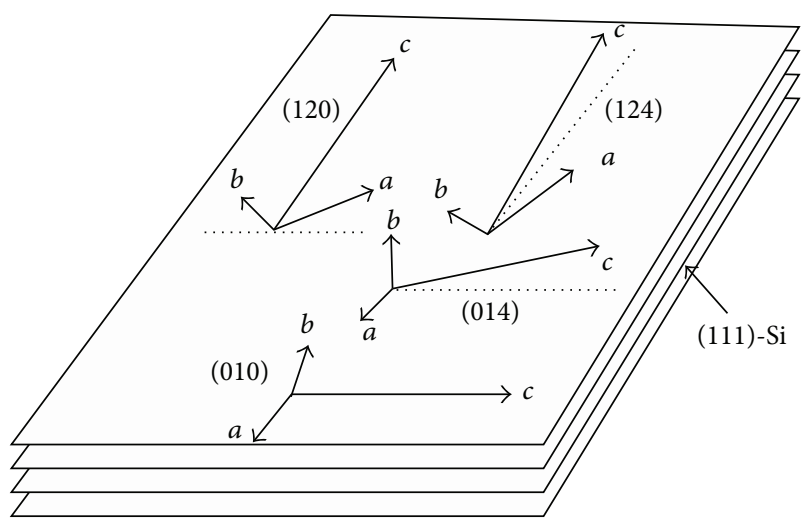

FIGURE 6: Sketch of a possible crystalline orientation of a textured film that might give the diffraction pattern observed experimentally, assuming the same structure as Kawamura et al.

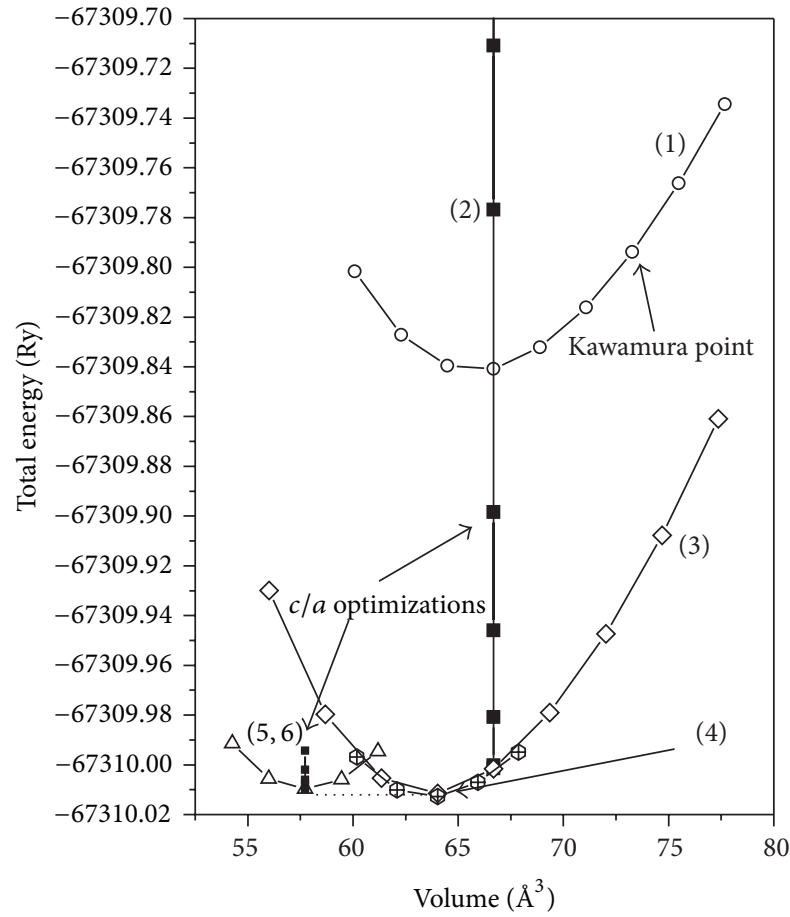

FIGURE 7: Trajectory of structural optimization of $\mathrm{ReN}_{2}$ by DFT.

space group is used. To avoid this, the structural degrees of freedom were increased by reducing the symmetry of the space group. Instead of the initial choice of P63/mmc (194), the Am2a (40) space group was used. Even using the lower-symmetry Am2a space group, the diffraction pattern generated is close to the pattern observed by Kawamura et al., as shown in Figure 8. More DFT refinements are recommended to improve the quality of the match.

\section{Conclusions}

From the experimental conditions, where a rhenium target was sputtered in nitrogen environments at different pressures, it can be concluded that incorporation of nitrogen causes

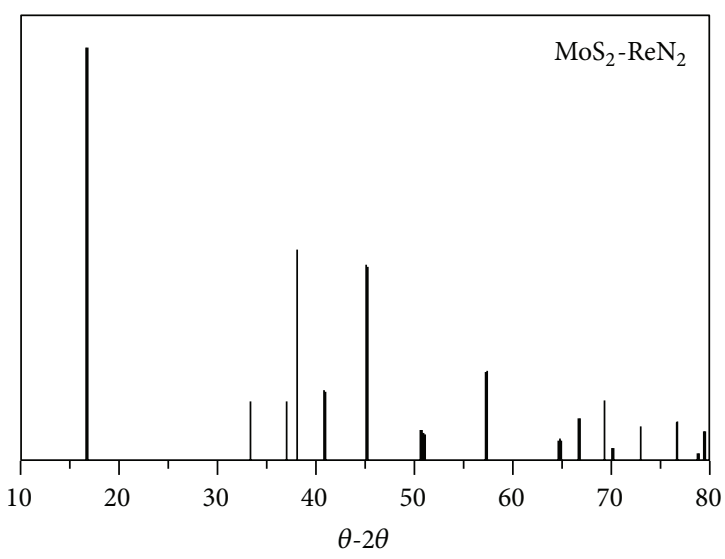

(a)

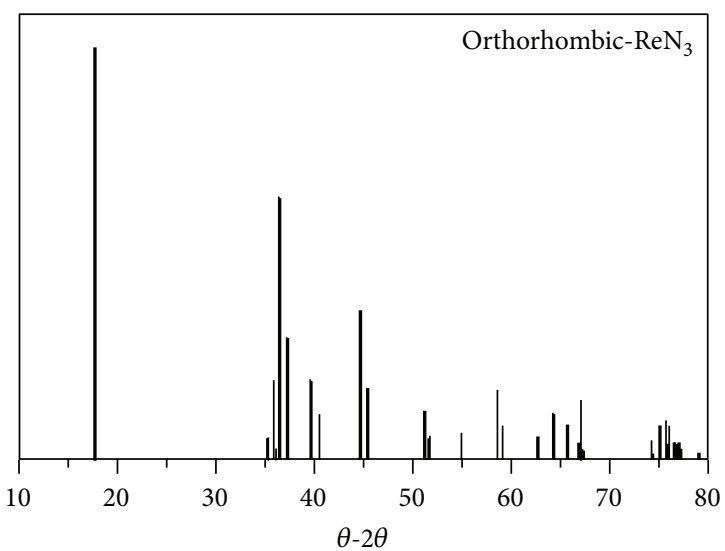

(b)

FIgURE 8: Comparison of the powder diffraction patterns of $\mathrm{ReN}_{2}$ and $\mathrm{ReN}_{3}$ using $\mathrm{Cu}-\mathrm{K}_{\alpha}$ radiation.

important expansion of the rhenium lattice, mainly in $c$ direction. The material synthesized by magnetron sputtering is similar to those prepared by the HPHT method, but with a different crystallographic texture. A diazenide group, as it was proposed by Kawamura, inserted within rhenium interstices could not explain the diffraction patterns of the recently prepared rhenium-nitrogen compounds. We propose that the azide group between the rhenium layers provides a better match for these diffraction patterns. The diffractograms given by DFT with $\mathrm{ReN}_{3}$ agree with the experimental evidence. Given that, the material should be $\mathrm{ReN}_{3}$ instead of $\mathrm{ReN}_{2}$. The $\mathrm{ReN}_{3}$ is a brittle material and also it is mechanically unstable under indentation. The possibility of forming of metal azides by sputtering deposition is a new field that must be further studied.

\section{Conflict of Interests}

The authors declare that there is no conflict of interests regarding the publication of this paper. 


\section{Acknowledgments}

The authors would like to give thanks to D. Dominguez for technical assistance. Financial support from DGAPAUNAM Grants IN114209, IT00314, and CONACYT 83275 is acknowledged. A. Reyes appreciates the sabbatical scholarship from PASPA-DGAPA-UNAM at Department of Physics, The Pennsylvania State University.

\section{References}

[1] E. Horvath-Bordon, R. Riedel, A. Zerr et al., "High-pressure chemistry of nitride-based materials," Chemical Society Reviews, vol. 35, no. 10, pp. 987-1014, 2006.

[2] J. C. Crowhurst, A. F. Goncharov, B. Sadigh et al., "Synthesis and characterization of the nitrides of platinum and iridium," Science, vol. 311, no. 5765, pp. 1275-1278, 2006.

[3] A. F. Young, C. Sanloup, E. Gregoryanz, S. Scandolo, R. J. Hemley, and H.-K. Mao, "Synthesis of novel transition metal nitrides $\mathrm{IrN}_{2}$ and $\mathrm{OsN}_{2}$," Physical Review Letters, vol. 96, no. 15, Article ID 155501, 2006.

[4] F. Kawamura, H. Yusa, and T. Taniguchi, "Synthesis of rhenium nitride crystals with $\mathrm{MoS}_{2}$ structure," Applied Physics Letters, vol. 100, no. 25, Article ID 251910, 2012.

[5] S. Vepřek, "The search for novel, superhard materials," Journal of Vacuum Science and Technology A, vol. 17, no. 5, pp. 2401-2420, 1999.

[6] A. P. Caricato, M. Fernàndez, G. Leggieri et al., "Reactive pulsed laser deposition of gold nitride thin films," Applied Surface Science, vol. 253, no. 19, pp. 8037-8040, 2007.

[7] G. Soto, "Synthesis of PtNx films by reactive laser ablation," Materials Letters, vol. 58, no. 16, pp. 2178-2180, 2004.

[8] M. G. Moreno-Armenta, J. Diaz, A. Martinez-Ruiz, and G. Soto, "Synthesis of cubic ruthenium nitride by reactive pulsed laser ablation," Journal of Physics and Chemistry of Solids, vol. 68, no. 10, pp. 1989-1994, 2007.

[9] G. Soto, A. Rosas, M. H. Farias, W. De la Cruz, and J. A. Diaz, "Characterization of rhenium nitride films produced by reactive pulsed laser deposition," Materials Characterization, vol. 58, no. 6, pp. 519-526, 2007.

[10] D. Güttler, B. Abendroth, R. Grötzschel, W. Möller, and D. Depla, "Mechanisms of target poisoning during magnetron sputtering as investigated by real-time in situ analysis and collisional computer simulation," Applied Physics Letters, vol. 85, no. 25, pp. 6134-6136, 2004.

[11] J. L. P. Blaha, K. Schwarz, G. Madsen, and D. Kvasnicka, Wien2k, an Augmented Plane Wave + Local Orbitals Program for Calculating Crystal Properties, Technische Universität Wien, Wien, Austria, 2001.

[12] J. P. Perdew, K. Burke, and M. Ernzerhof, "Generalized gradient approximation made simple," Physical Review Letters, vol. 77, no. 18 , pp. 3865-3868, 1996.

[13] C. Stampfl, W. Mannstadt, R. Asahi, and A. J. Freeman, "Electronic structure and physical properties of early transition metal mononitrides: density-functional theory LDA, GGA, and screened-exchange LDA FLAPW calculations," Physical Review $B$, vol. 63, no. 15, Article ID 155106, 11 pages, 2001.

[14] A. B. Gordienko, Y. N. Zhuravlev, and A. S. Poplavnoi, "Energy band structure of silver azide $\left(\mathrm{AgN}_{3}\right)$," Soviet Physics Journal, vol. 35, no. 2, pp. 130-132, 1992.
[15] G. Soto, H. Tiznado, A. Reyes, and W. De La Cruz, "First principles calculations of interstitial and lamellar rhenium nitrides," Journal of Alloys and Compounds, vol. 514, pp. 127-134, 2012.

[16] M. Fuchigami, K. Inumaru, and S. Yamanaka, "Interstitial binary nitride ReNx phases prepared by pulsed laser deposition: structure and superconductivity dependence on nitrogen stoichiometry," Journal of Alloys and Compounds, vol. 486, no. 1-2, pp. 621-627, 2009.

[17] A. C. Fischer-Cripps, Nanoindentation, Springer, New York, NY, USA, 2002.

[18] B. Moser, J. Kuebler, H. Meinhard, W. Muster, and J. Michler, "Observation of instabilities during plastic deformation by in-situ SEM indentation experiments," Advanced Engineering Materials, vol. 7, no. 5, pp. 388-392, 2005.

[19] C. A. Schuh, "Nanoindentation studies of materials," Materials Today, vol. 9, no. 5, pp. 32-40, 2006.

[20] G. Soto, "Computational study of Hf, Ta, W, Re, Ir, Os and Pt pernitrides," Computational Materials Science, vol. 61, pp. 1-5, 2012.

[21] X. P. Du, Y. X. Wang, and V. C. Lo, "Investigation of tetragonal $\mathrm{ReN}_{2}$ and $\mathrm{WN}_{2}$ with high shear moduli from first-principles calculations," Physics Letters A, vol. 374, no. 25, pp. 2569-2574, 2010.

[22] E. Zhao and Z. Wu, "Structural, electronic and mechanical properties of $\mathrm{ReN}_{2}$ from first principles," Computational Materials Science, vol. 44, no. 2, pp. 531-535, 2008.

[23] Y. Wang, T. Yao, J.-L. Yao, J. Zhang, and H. Gou, "Does the real $\mathrm{ReN}_{2}$ have the $\mathrm{MoS}_{2}$ structure?" Physical Chemistry Chemical Physics, vol. 15, no. 1, pp. 183-187, 2013. 

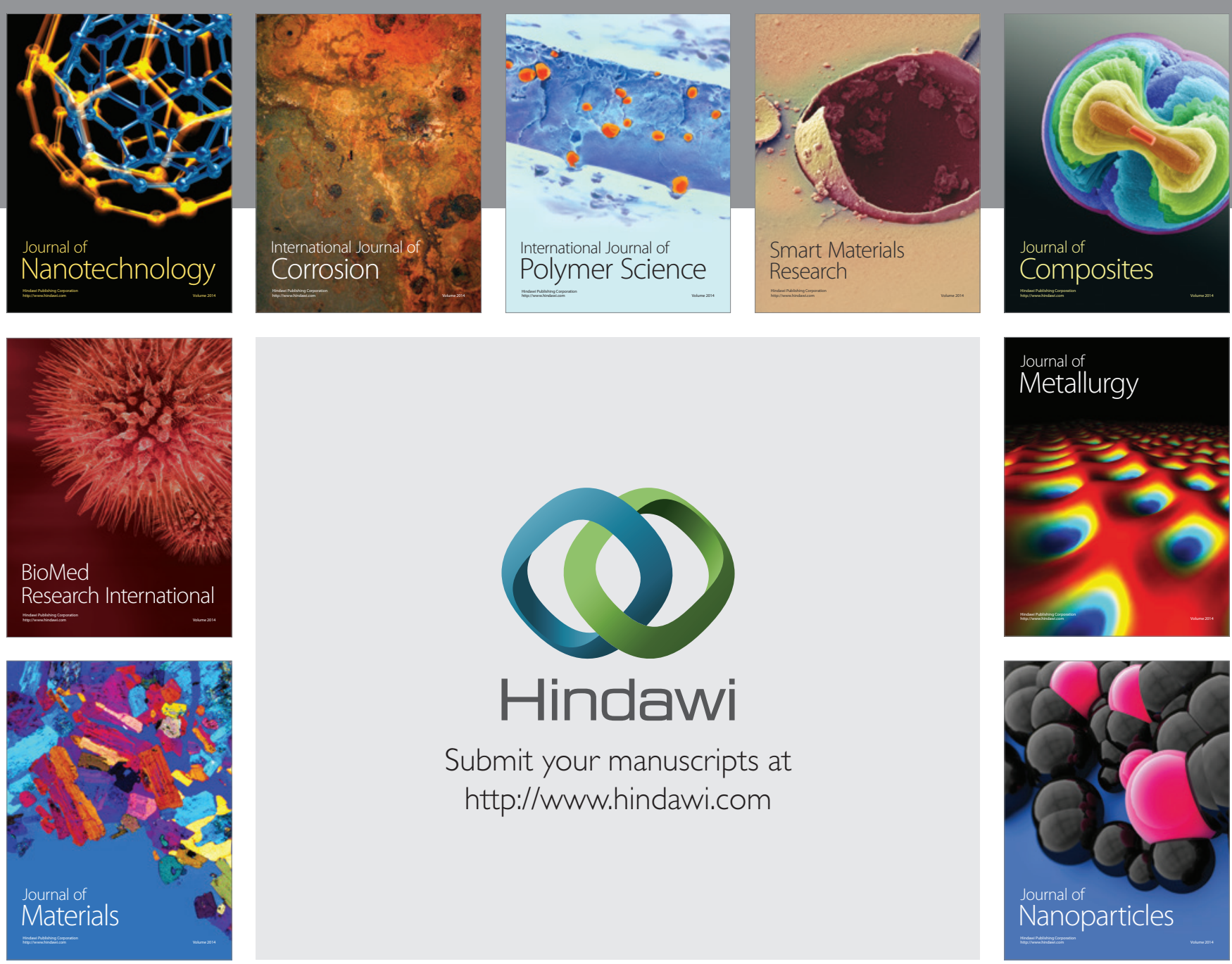

Submit your manuscripts at http://www.hindawi.com
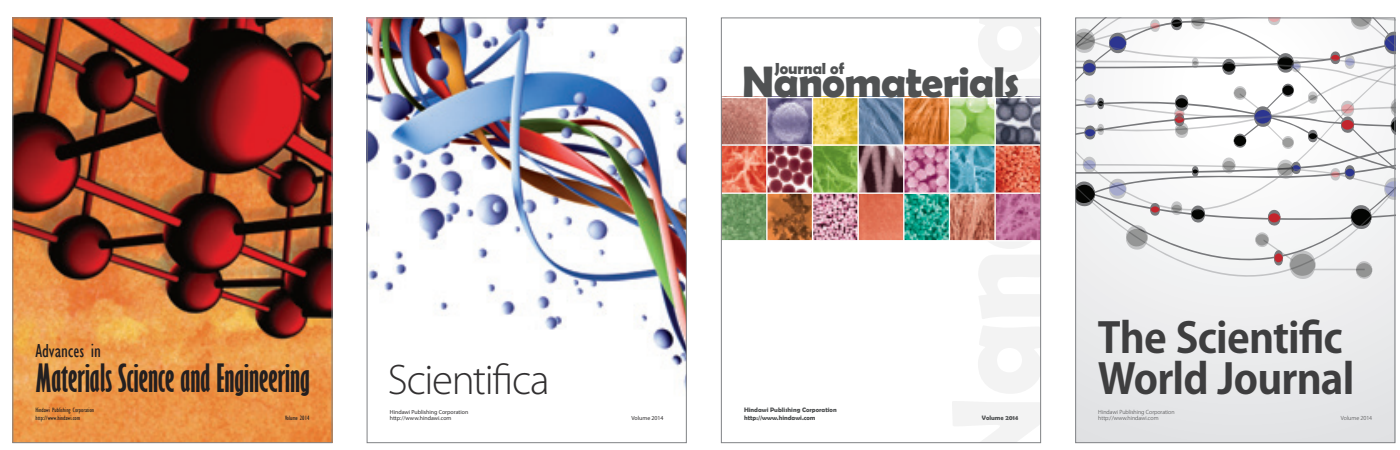

\section{The Scientific World Journal}
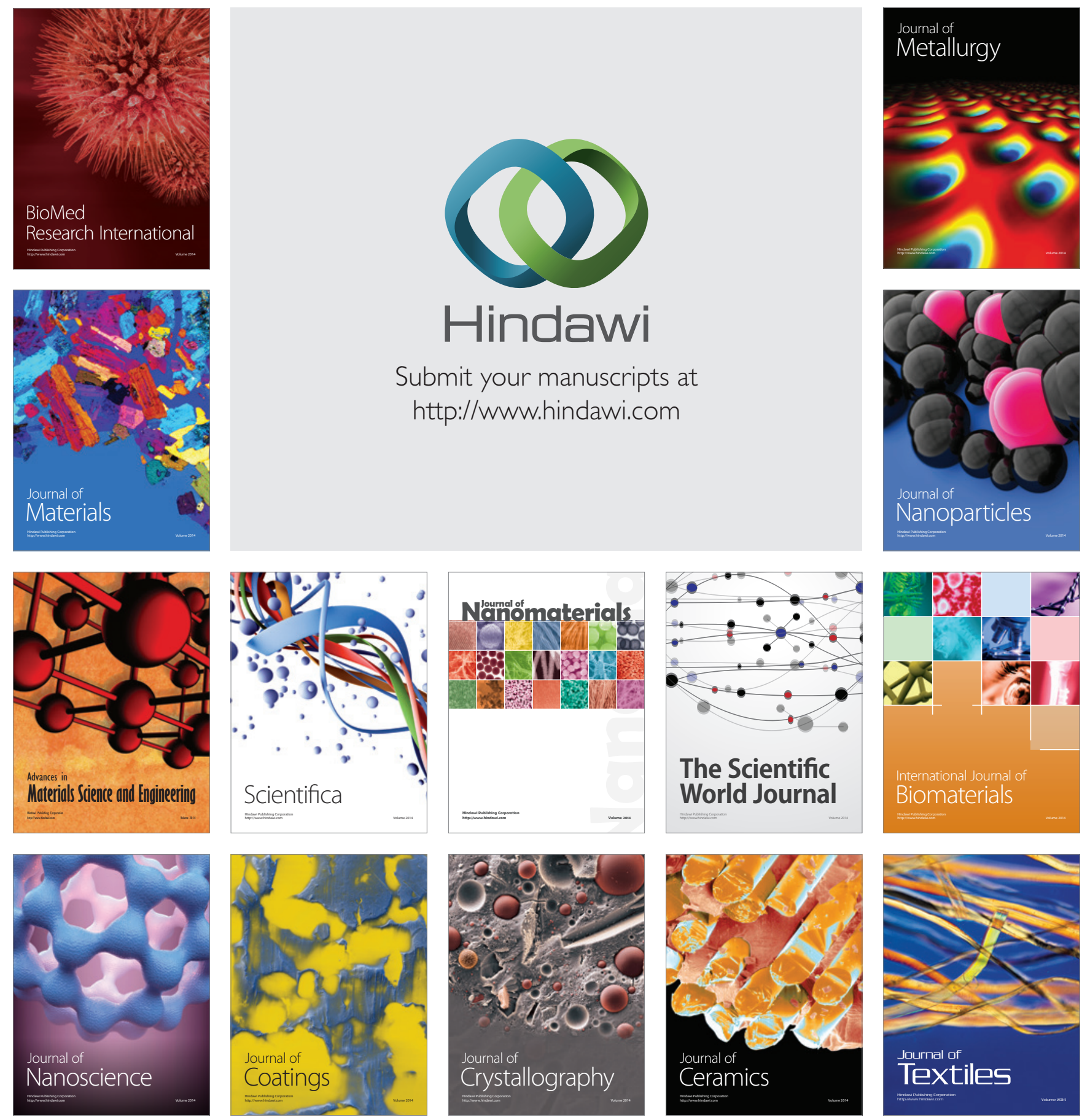